\title{
Diltiazem Prophylaxis for the Prevention of Atrial Fibrillation in Patients Undergoing Thoracoabdominal Esophagectomy: A Retrospective Cohort Study
}

\author{
Marcel Hochreiter ${ }^{1,2}$ - Thomas Schmidt ${ }^{3}$ Benedikt H. Siegler ${ }^{2}$ Leila Sisic ${ }^{3}$ • \\ Karsten Schmidt $^{1,2}$ - Thomas Bruckner ${ }^{4}$ - Beat P. Müller-Stich ${ }^{3}$ - Markus K. Diener ${ }^{3}$. \\ Markus A. Weigand ${ }^{2} \cdot$ Markus W. Büchler ${ }^{3}$. Cornelius J. Busch ${ }^{2}$
}

Published online: 4 March 2020

(C) The Author(s) 2020

\begin{abstract}
Background Atrial fibrillation (AF) represents the most frequent arrhythmic disorder after thoracoabdominal esophageal resection and is associated with a significant increase in perioperative morbidity and mortality.

Methods In this retrospective cohort study, 167 patients who underwent thoracoabdominal esophagectomy at a large university hospital were assessed. We compared patients who received a 14-day postoperative course of diltiazem with a control group of patients who did not undergo diltiazem prophylaxis. Diltiazem therapy started immediately upon admission to the intensive care unit (ICU) with a loading dose of $0.25 \mathrm{mg} / \mathrm{kg}$ bodyweight (i.v.) followed by continuous infusion $\left(0.1 \mathrm{mg} / \mathrm{kg}\right.$ bodyweight $/ \mathrm{h}$ ) for $40-48 \mathrm{~h}$. Oral administration (Dilzem ${ }^{\circledR} 180 \mathrm{mg}$ uno retard, once a day) was started on postoperative day 3 .

Results A total of 117 patients were assessed. Twelve (10.3\%) of all patients developed postoperative new-onset atrial fibrillation in the first 30 days after surgical intervention. Prevalence of new-onset AF showed no significant differences between the diltiazem group and control group $(p=0.74)$. The prevalence of bradycardia $(14.7 \%$ vs. $3.6 \% ; p=0.03)$ and dose of norepinephrine required ( $0.09 \mathrm{vs} .0 .04 \mu \mathrm{g} / \mathrm{kg}$ bodyweight $/ \mathrm{min} ; p=0.04)$ were higher in the diltiazem group. There were no significant differences between the groups for the median postoperative duration of hospital/ICU stay or mortality.

Conclusions A prophylactic 14-day postoperative course of diltiazem was not associated with a reduction in newonset AF or 30-day mortality following thoracoabdominal esophagectomy. Prophylactic diltiazem therapy was associated with drug-related adverse effects such as bradycardia and increased requirement of norepinephrine.

German Clinical Trial Registration Number: DKRS00016631.
\end{abstract}

Cornelius J. Busch

cornelius.busch@med.uni-heidelberg.de

1 Department of Anesthesiology and Intensive Care Medicine, Essen University Hospital, Essen, Germany

2 Department of Anesthesiology and Intensive Care Medicine, Heidelberg University Hospital, Heidelberg, Germany

3 Department of General, Visceral and Transplantation Surgery, Heidelberg University Hospital, Heidelberg, Germany

4 Institute of Medical Biometry and Informatics, Heidelberg University Hospital, Heidelberg, Germany

\section{Introduction}

Postoperative atrial fibrillation (POAF) is the most common cardiac arrhythmia after esophagectomy and has been reported to appear in $\leq 44 \%$ of patients [1,2]. Although POAF in thoracic surgery has been considered to be temporary in most cases [3], its occurrence in the postoperative phase is associated with a substantial increase in morbidity, mortality, resource utilization and long-term risk of stroke [2, 4-6]. Direct results of POAF include a decrease in cardiac output and development of atrial thrombosis. The etiology of POAF is incompletely understood. 
Development of POAF requires vulnerable atrial tissue and a trigger to initiate AF [7]. The risk factors for POAF are being male, age $>75$ years, nicotine/alcohol consumption, cardiopulmonary comorbidities (i.e., arterial hypertension, chronic obstructive pulmonary disease, congestive heart failure), preoperative episode of $\mathrm{AF}$, as well as the location (irritation of atria) and magnitude of surgery (including esophagectomy) [8-10]. Surgical procedures are associated with local or systemic inflammation affecting the vulnerability of the atrial substrate to POAF [11]. Usually, AF onset occurs on postoperative day (POD)2 and POD3 [12], whereas the risk of arrhythmias decreases over the first postoperative month to that before surgery independent of the treatment given [13].

POAF represents a major (but potentially preventable) adverse outcome. For thoracic surgical procedures, current recommendations from the American Association for Thoracic Surgery Taskforce favor prevention strategies based on pharmacology [7]. In detail: (1) oral beta-blockers should be continued after surgery to avoid withdrawal; (2) all patients should receive magnesium (i.v.) perioperatively if the serum magnesium level is low; (3) for most patients at an increased risk of POAF development, preventive administration of diltiazem or amiodarone (especially for esophagectomy) may be reasonable [7]. Undoubtedly, amiodarone is among the most efficacious antiarrhythmic agents, but its use is associated with potentially serious toxicity, so in our institution diltiazem is used for patients with preserved cardiac function for PAOF prevention [14].

Based on several double-blinded controlled trials in cardiac surgery and lung surgery, the efficacy of calcium channel blockers has been tested for the prophylaxis of POAF [15-17]. In those studies, the prevalence of POAF was reduced by $\sim 50 \%$ [18]. Diltiazem use is associated with a far lower prevalence of hypotension than verapamil use, so diltiazem is the recommended calcium blocking agent. Diltiazem inhibits L-type calcium channels in vascular and conduction tissue, and especially in nodal tissue [19].

Despite the demonstrable prophylactic effect of diltiazem against POAF in cardiac surgery and lung surgery, data regarding its efficacy in the prevention of POAF in patients undergoing esophagectomy are lacking. Therefore, we evaluated the effect of 14-day postoperative prophylaxis with the calcium antagonist diltiazem to prevent POAF in patients undergoing thoracoabdominal esophagectomy.

\section{Patients and methods}

\section{Ethical approval of the study protocol}

The present study was undertaken in accordance with the 1975 Helsinki Declaration and its later amendments and after approval from the ethics committee of the Medical Faculty of Heidelberg University (Heidelberg, Germany; $\mathrm{S}-493 / 2016)$. Due to its retrospective character, written informed consent was not needed.

\section{Data collection}

A retrospective cohort study was conducted to evaluate the efficacy and safety of postoperative administration of diltiazem in patients undergoing thoracoabdominal esophagectomy at Heidelberg University Hospital. Patients were identified from an internal clinical prospectively maintained database. Electronic medical records were reviewed for demographic and clinical characteristics, concomitant diseases, medications, surgery type, laboratory values, occurrence of POAF, as well as the prevalence and nature of postoperative adverse events and complications.

\section{Outcome measures}

The primary outcome measure was the prevalence of newonset AF lasting $\geq 30$ s or for the duration of the electrocardiography recording (if $<30 \mathrm{~s}$ ) within 30 days after thoracoabdominal esophagectomy. Secondary outcome measures were duration of postoperative stay in hospital or intensive care unit (ICU), prevalence of adverse events, as well as 30-day and 90-day mortality. Bradycardia was defined as heart rate $<50$ beats per minute. Hypotension was defined as systolic blood pressure $<90 \mathrm{mmHg}$ that necessitated administration of fluid/and or vasopressors.

\section{Study population and surgical procedure}

All patients aged $\geq 18$ years who underwent thoracoabdominal esophagectomy with abdominal and mediastinal lymphadenectomy for adenocarcinoma or squamous cell carcinoma between 2011 and 2015 were included in data assessment and screened for eligibility from a prospectively maintained database. Thoracoabdominal esophagectomy was undertaken via laparotomy followed by right-sided thoracotomy and intrathoracic anastomosis (Ivor Lewis procedure) [20]. Patients with incomplete data sets, disturbances of sinus functions, second- and thirddegree atrioventricular blocks, who were taking antiarrhythmic drugs preoperatively or with contraindications against diltiazem prophylaxis (i.e., medication with $\beta$-receptor antagonists, reduced cardiac function) were excluded from the final analysis (Fig. 1). 


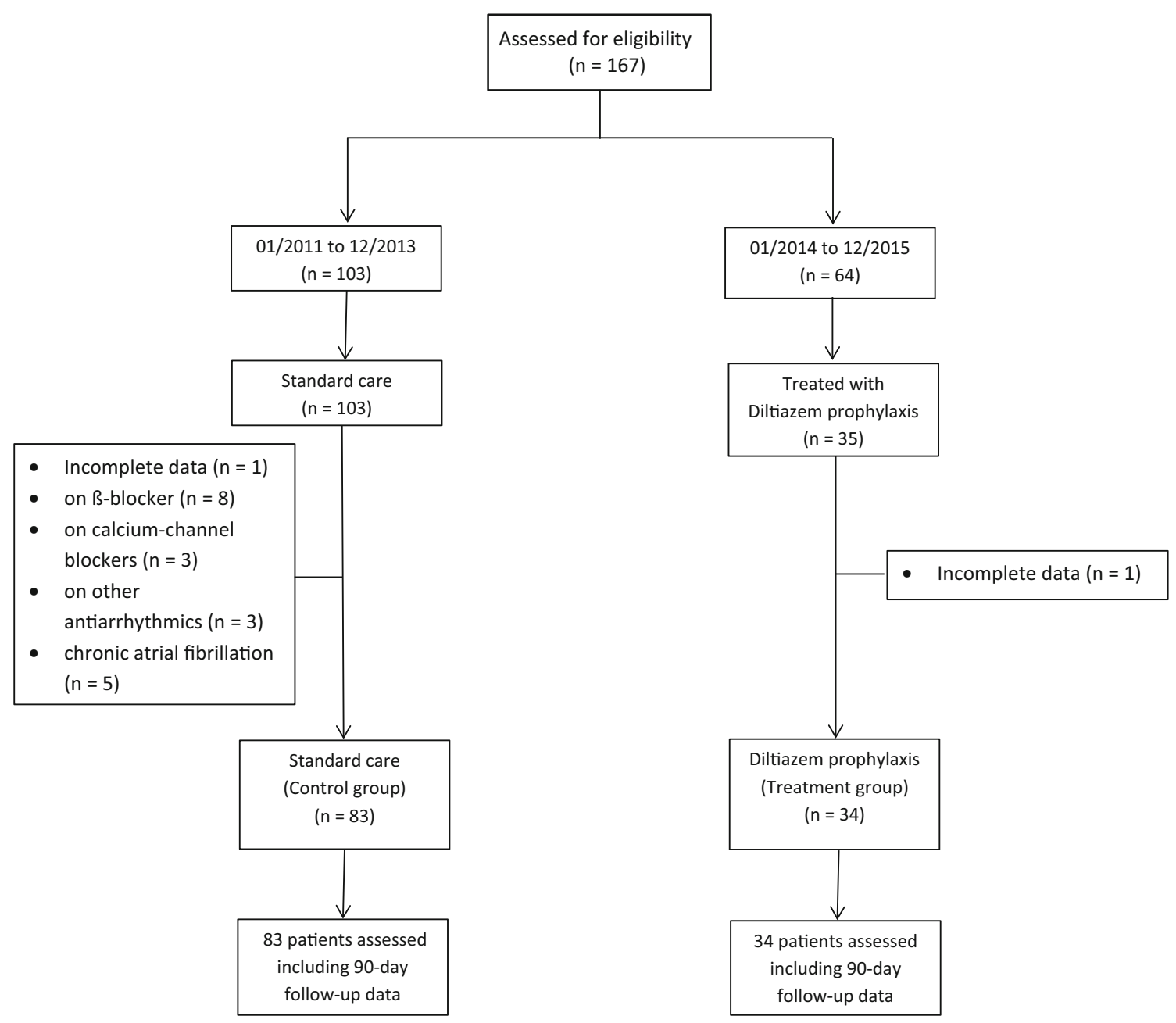

Fig. 1 Flowchart of the study enrollment population

\section{Perioperative care and postoperative diltiazem prophylaxis}

After surgery, patients were admitted routinely to the ICU. In accordance with evidence for prevention of $\mathrm{AF}$ related to general thoracic surgery [7], from 2014 onwards diltiazem prophylaxis was conducted routinely in patients undergoing thoracoabdominal esophagectomy. In accordance with the local ICU protocol, contraindications for diltiazem were reduced cardiac function, disturbances of sinus functions, second- and third-degree atrioventricular blocks, or treatment with antiarrhythmic drugs before surgery.

Accordingly, patients included in the present study were assigned to the control group (before 2014) or treatment group (after 2014). Patients in both study groups were treated identically with regard to surgical procedure, perioperative administration of fluids, enteral feeding, and drainage management. In accordance with the local ICU protocol: (1) the serum levels of potassium ions $\left(\mathrm{K}^{+}\right)$were maintained at the high end of normal (4.5-5.5 mEq/L); (2) in the event of clinical deterioration computed tomography was undertaken (including evidence of infection).

In the treatment group, diltiazem prophylaxis was given as per the local ICU protocol for 14 days. Therapy started immediately upon ICU admission with a loading dose of diltiazem $(0.25 \mathrm{mg} / \mathrm{kg}$ bodyweight, i.v. $)$ given over $30 \mathrm{~min}$, followed by continuous infusion $(0.1 \mathrm{mg} / \mathrm{kg}$ bodyweight $/ \mathrm{h}$ ) for $40-48 \mathrm{~h}$. Administration was discontinued if the pulse or systolic blood pressure dropped below 60 beats per min or $90 \mathrm{mmHg}$, respectively. Oral administration (Dilzem ${ }^{\circledR} 180$ uno retard, once a day) started at POD3 when patients were relocated to the normal ward. All patients underwent continuous telemetry monitoring until POD14. After POD14, vital signs were documented three times a day until the patient was discharged from hospital, and a 12-lead ECG was taken (if appropriate). 


\section{Statistical analyses}

Descriptive statistics for empirical distributions are the mean \pm SD (continuous data) and absolute and relative frequencies (categorical data). Possible differences between patient groups were evaluated using the MannWhitney $U$-test for continuous variables and Chi-square test or Fisher's exact test (where appropriate) for categorical variables. The $95 \%$ confidence interval (CI) of the difference was calculated. $p<0.05$ was considered significant. Analyses were performed using SASTM 9.4 (SAS Institute, Cary, NC, USA).

\section{Results}

\section{Patient characteristics}

In total, between 2011 and 2015, 167 patients underwent thoracoabdominal esophagectomy at our institution. Fifty patients were excluded from the final data analysis, mainly because of chronic AF or because they were already on Bblockers or calcium channel blockers as background therapy (Fig. 1). Of the remaining 117 patients, 34 individuals (29\%) received diltiazem prophylaxis, whereas 83 (71\%) did not. In both groups, the duration of follow-up was 90 days. Both groups were comparable in terms of age, sex, body mass index, underlying disease and preoperative treatment. Table 1 summarizes the baseline characteristics of all included patients.

\section{Perioperative management and surgical outcome}

Data for intraoperative management and postoperative outcome are listed in Tables 2 and 3, respectively. Most patients received combined epidural and general anesthesia (control group, 81.9\%; treatment group, 91.2\%; $p=0.21$ ). The duration of the surgical procedure, blood loss, as well as the intraoperative volume of fluids and transfused blood, were comparable between the groups. One patient in the treatment group (2.9\%) and two patients in the control group (2.4\%) needed continuous veno-venous hemofiltration after surgery $(p=0.87)$. There was no significant difference between the groups in the prevalence of postoperative complications, such as sepsis $(p=0.40)$, anastomotic leakage $(p=0.41)$, pneumonia $(p=0.50)$ or requirement of respiratory support $(p=0.95)$. No POAF patients were diagnosed with cardioembolic stroke during hospital stay.

\section{Postoperative prevalence of AF and need for cardio- circulatory medication}

Overall, POAF occurred in 12 patients after esophagectomy (10.3\%) (Table 3). Most patients (10/12, 83.3\%) developed POAF within $72 \mathrm{~h}$ of surgery. The proportion of patients with POAF did not differ significantly between the control group and individuals with postoperative diltiazem prophylaxis (difference $-2 \%$; 95\% CI -13.1 to 13.8 ; $p=0.74)$. No significant differences were found in the number of patients requiring postoperative administration of catecholamines $(p=0.09)$, including dobutamine $(p=0.89)$. Patients in the diltiazem group received postoperative higher doses of norepinephrine ( 0.09 vs. $0.04 \mu \mathrm{g}$ /

Table 1 Characteristics of the study cohort

\begin{tabular}{llll}
\hline & $\begin{array}{l}\text { Diltiazem } \\
(n=34)\end{array}$ & $\begin{array}{l}\text { Control } \\
(n=83)\end{array}$ \\
\hline $\begin{array}{l}\text { Age (years) } \\
\text { Sex }\end{array}$ & $60.0( \pm 9.8)$ & $60.9( \pm 11.8)$ & 0.188 \\
Male & $30(88.2)$ & $62(74.7)$ & 0.105 \\
Female & $4(11.8)$ & $21(25.3)$ \\
BMI $\left(\mathrm{kg} / \mathrm{m}^{2}\right)$ & $23.8( \pm 3.9)$ & $25.2( \pm 4.6)$ & 0.124 \\
Diabetes mellitus & $4(11.8)$ & $4(4.8)$ & 0.177 \\
Arterial hypertension & $14(41.2)$ & $28(33.7)$ & $8(9.6)$ \\
Coronary heart disease & $2(5.9)$ & $11(13.2)$ & 0.446 \\
COPD & $4(11.8)$ & $1(1.2)$ & 0.509 \\
Renal insufficiency & $1(2.9)$ & $68(81.9)$ \\
Preoperative chemotherapy and/or radiotherapy & $23(67.6)$ & 0.827 \\
\hline
\end{tabular}

Data are the mean \pm standard deviation or number $(\%)$

$B M I$ body mass index, $C O P D$ chronic obstructive pulmonary disease 
Table 2 Intraoperative data

\begin{tabular}{|c|c|c|c|c|}
\hline & $\begin{array}{l}\text { Diltiazem } \\
(n=34)\end{array}$ & $\begin{array}{l}\text { Control } \\
(n=83)\end{array}$ & $\begin{array}{l}\text { Difference } \\
(95 \% \text { CI })\end{array}$ & $p$ \\
\hline Duration of surgery (min) & $266( \pm 62)$ & $259( \pm 110)$ & $7.0(-25.1$ to 39.1$)$ & 0.728 \\
\hline Intraoperative fluid administration (mL) & $3610( \pm 1028)$ & $3610( \pm 1375)$ & $0.1(-462$ to 462.0$)$ & $>0.999$ \\
\hline Blood loss (mL) & $763( \pm 382)$ & $832( \pm 514)$ & $-69(-241$ to 103$)$ & 0.483 \\
\hline Units of blood transfused & $0.41( \pm 1.08)$ & $0.72( \pm 1.60)$ & $-0.31(-0.82$ to 0.20$)$ & 0.301 \\
\hline Need for a thoracic epidural catheter & $31(91.2)$ & $68(81.9)$ & $9.3 \%(-7.1$ to 21.4$)$ & 0.208 \\
\hline
\end{tabular}

Data are the mean \pm standard deviation or number $(\%)$

CI confidence interval

Table 3 Postoperative outcome

\begin{tabular}{|c|c|c|c|c|}
\hline & $\begin{array}{l}\text { Diltiazem } \\
(n=34)\end{array}$ & $\begin{array}{l}\text { Control } \\
(n=83)\end{array}$ & $\begin{array}{l}\text { Difference } \\
(95 \% \text { CI })\end{array}$ & $p$ \\
\hline \multicolumn{5}{|l|}{ Cardiovascular system } \\
\hline Hypotension* & $14(41.2)$ & $31(37.4)$ & $3.8 \%(-15.3$ to 24.1$)$ & 0.699 \\
\hline Bradycardia** & $5(14.7)$ & $3(3.6)$ & $11.1 \%(-27.5$ to 0.0$)$ & 0.031 \\
\hline Need for catecholamines & $19(55.9)$ & $32(38.6)$ & $17.3 \%(-3.5$ to 36.9$)$ & 0.086 \\
\hline Norepinephrin ( $\mu \mathrm{g} / \mathrm{kg} / \mathrm{min})$ & $0.09( \pm 0.13)$ & $0.04( \pm 0.07)$ & $0.05(0.00$ to 0.10$)$ & 0.041 \\
\hline Dobutamin $(\mu \mathrm{g} / \mathrm{kg} / \mathrm{min})$ & $0.76( \pm 1.39)$ & $0.76( \pm 1.88)$ & $-0.05(-0.68$ to 0.59$)$ & 0.888 \\
\hline Atrial fibrillation (new onset) & $3(8.8)$ & $9(10.8)$ & $-2.0 \%(-13.1$ to 13.8$)$ & 0.744 \\
\hline Atrial fibrillation (new onset) as a solitary complication & $1(2.9)$ & $3(3.6)$ & $-0.7 \%(-8.2$ to 12.2$)$ & 0.856 \\
\hline \multicolumn{5}{|l|}{ Respiratory system } \\
\hline Duration of respiratory support in the ICU $(\mathrm{h})^{* * *}$ & $24.9( \pm 80.4)$ & $26.2( \pm 100.0)$ & $-1.3(-37.2$ to 34.5$)$ & 0.946 \\
\hline Pneumonia & $3(8.8)$ & $11(13.2)$ & $-4.4 \%(-16.0$ to 11.5$)$ & 0.503 \\
\hline \multicolumn{5}{|l|}{ Other } \\
\hline CVVH & $1(2.9)$ & $2(2.4)$ & $0.5 \%(-6.3$ to 13.5$)$ & 0.869 \\
\hline Sepsis & $2(5.9)$ & $9(10.8)$ & $-4.9 \%(-15.3$ to 9.7$)$ & 0.404 \\
\hline Anastomotic leakage & $3(8.8)$ & $12(14.4)$ & $-5.6 \%(-17.4$ to 10.3$)$ & 0.408 \\
\hline \multicolumn{5}{|l|}{ Mortality } \\
\hline 30 days & $3(8.8)$ & $4(4.8)$ & $4.0 \%(-5.5$ to 19.4$)$ & 0.407 \\
\hline 90 days & $3(8.8)$ & $5(6.0)$ & $2.8 \%(-7.1$ to 18.5$)$ & 0.586 \\
\hline
\end{tabular}

Data are the mean \pm standard deviation or number $(\%)$

$C I$ confidence interval, $I C U$ intensive care unit, $C V V H$ continuous veno-venous hemofiltration

*Systolic pressure $<90 \mathrm{mmHg}, * *$ heart rate $<50$ beats/min, ***ventilation and noninvasive ventilation

$\mathrm{kg}$ bodyweight/min, $p=0.04$ ) in the ICU (Table 3). The prevalence of bradycardia was higher in the diltiazem group than in the control group ( $p=0.03)$. Five $(14.7 \%)$ of diltiazem-treated patients required temporary discontinuation of the drug because of bradycardia and hypotension.

POAF was frequently associated with one or more other complications. The most common surgical complication associated with POAF was anastomotic leakage. POAF and anastomotic leakage occurred in 8 of 12 patients. Four of them had additional pulmonary complications. POAF presented as a solitary complication in 4 of 12 patients. It took on average 3 days after the onset of POAF to diagnose anastomotic leakage, whereas pulmonary complications preceded the onset of POAF. Neither 30-day nor 90-day mortality differed significantly between the control group and diltiazem group $(p=0.41$ and $p=0.59)$ (Table 3).

\section{Duration of postoperative stay in hospital or ICU}

The median postoperative duration of hospital stay and ICU stay in the overall study cohort $(n=117)$ was 15 days (interquartile range [IQR], 13-20 days) and 2 (IQR, 1-4 days), respectively. There was no significant difference in the median duration of postoperative hospital stay 
in the diltiazem group compared with that in the control group (14 days, IQR $12-19$ vs. 15 days, IQR 13-21 days; $p=0.15$ ) nor in the median duration of ICU stay between the two groups ( 2 days, IQR $1-5$ vs. 2 days, IQR $1-4$ days, $p=0.35$ ).

\section{Discussion}

Esophagus resection through a thoracoabdominal approach is the first-line treatment for esophagus carcinoma [21, 22]. Besides surgical complications [23] and pulmonary infection [24], AF is one of the most common complications and is associated with a substantial increase in morbidity, mortality, resource utilization and long-term risk of stroke. Reducing the prevalence of POAF in esophagectomy is extremely important.

We showed that postoperative antiarrhythmic prophylaxis with diltiazem for 14 days did not reduce the prevalence of new-onset AF in the first 30 days after surgery and did not influence mortality after thoracoabdominal esophagectomy.

Patients who have a thoracoabdominal esophagectomy are at a high risk of developing new-onset $\mathrm{AF}$, and a range from 10 to $44 \%$ has been reported $[8,25,26]$. The overall prevalence of POAF observed in the present study was $10.3 \%$, which was lower than expected. However, it is difficult to compare data between studies without taking risk factors into account. A consistent clinical predictor of POAF after major thoracic procedures is older age $[12,26]$. Aging causes degenerative changes in atrial anatomy, including dilation and fibrosis, which results in a vulnerable atrial myocardium [27]. This is accompanied by shorter atrial effective refractoriness, longer sinoatrial and nodal conduction times, atrial stiffening and splitting of the atrial excitation waveform caused by the pectinated trabeculae [15]. Lohani et al. [28] showed that patients aged $>65$ years experienced a higher prevalence of POAF after esophagectomy. In the present study, the mean age was 60.4 years, which might have lowered the risk of developing POAF.

A further important risk factor for POAF is the degree of surgical stress, which causes systemic and local inflammation. Okamura et al. [29] showed that, in patients after esophagectomy, the longer the surgical procedure, the higher the release of proinflammatory cytokines in serum (e.g., interleukin (IL)-6 and IL-8). Subsequently, a metaanalysis demonstrated that an increase in circulating levels of proinflammatory factors was associated with a greater risk of POAF in the general population as well as in patients who underwent coronary artery bypass grafting [30]. The median duration of surgery in the present study was $261 \mathrm{~min}$, which is shorter compared with the data from a study by Tisdale et al. [31], who reported a median duration of surgery of $477.5 \mathrm{~min}$ and prevalence of POAF of $40 \%$. However, some cases of subclinical and transient POAF may have been missed because of less intensive cardiac monitoring on the normal ward after POD14 during hospitalization.

The high prevalence of POAF after major thoracic surgery has led to several studies investigating the role of postoperative pharmacologic prophylaxis $[16,31,32]$. However, much of the evidence has been extrapolated from cardiac surgery studies. Only a few trials have been conducted exclusively in patients undergoing esophagectomy $[25,33,34]$. Tisdale et al. evaluated, in one randomized controlled study $(n=80)$ and very recently in a retrospective cohort study $(n=220)$, the effect of amiodarone on POAF with esophagectomy and found fewer patients with POAF in the amiodarone group. The randomized controlled study did not find a difference in other parameters between the two groups, but the cohort study revealed that amiodarone (i.v.) was associated with hypotension, bradycardia and corrected prolongation of the QT interval [25, 34]. However, due to potential side effects such as lung toxicity (probably only in high-dose therapy), amiodarone is not used widely in clinical practice for this indication. Ojima et al. investigated landiolol use in patients after esophagectomy. AF occurred in 30\% of cases, whereas perioperative $\beta$-blockade reduced it to $10 \%$ [33]. Prophylactic use of $\beta$-blockers in the perioperative setting is controversial. Studies in noncardiac thoracic surgery have shown a high prevalence of hypotension and bradycardia [32, 35]. In 2014, guidelines set by the American Association for Thoracic Surgery did not recommend prophylactic use of $\beta$-blockers for POAF prevention unless the patient was already on $\beta$-blocker therapy before surgery to avoid withdrawal [7].

Previously, prophylactic use of diltiazem showed promising results in reducing POAF after lung surgery. In two prospective randomized trials, Amar et al. reported significant reduction in POAF compared with that elicited by placebo or digoxin $[15,36]$. We used the same dose regimen in patients after esophagus resection. Interestingly, our data showed a similar prevalence of POAF in patients with diltiazem prophylaxis compared with those receiving standard care. More side effects (e.g., hypotension, bradycardia) were found in patients receiving diltiazem. Subsequent administration of diltiazem was associated with significantly higher doses of norepinephrine. This strategy is potentially harmful because during esophagectomy, multiple arteries are ligated and the newly formed gastric tube is dependent only on the right gastro-epiploic artery. Fumagalli et al. [37] showed in a observational study that hypotensive episodes (decrease in systolic pressure $>30 \%$ of the baseline value for $>5 \mathrm{~min}$ ) and use 
of vasopressors worsened local perfusion and were the main contributing factors for anastomotic leaks in patients undergoing esophagectomy. However, our data showed a similar prevalence of anastomotic leaks in both groups.

Bradycardia is a common side effect of diltiazem treatment due to inhibition of L-type calcium channels in sinus nodes. This negative chronotropic effect was also observed in the diltiazem group of the present study. These data are in accordance with results from other studies because patients with ischemic heart disease showed lower heart rates starting at $0.25 \mathrm{mg} / \mathrm{kg}$ (i.v.) [38, 39], which is equivalent to the initial loading dose. Another possible mechanism of bradycardia might be thoracic epidural anesthesia via blockade of cardiac rami [40], but this was not evident in our data.

Embolic stroke is the most feared complication of POAF. Gialdini et al. evaluated stroke risk due to POAF from a database including $>1$ million noncardiac surgical patients. They documented a $1.47 \%$ cumulative risk of stroke 1 year after hospitalization, compared with $0.36 \%$ in patients who did not develop POAF [6]. Anticoagulation is the cornerstone of prevention of embolic stoke, but it is challenging in the perioperative period. Postoperative patients carry a higher risk of bleeding and a hypercoagulable state simultaneously. American and European guidelines state that it is reasonable to administer antithrombotic medication if POAF persists for $>48 \mathrm{~h}$, but this is not a specific recommendation for noncardiac surgery patients because direct evidence is not available [41, 42].

We demonstrated that anastomotic leakage was a frequent morbidity in patients with POAF. Eight out of 12 patients $(66.7 \%)$ with POAF showed anastomotic leakage. This value is consistent with a recent study by Sessing et al. [43] in which $77.4 \%$ of patients with POAF after esophagectomy had infectious complications such as pneumonia $(41.9 \%)$ and anastomotic leakage or conduit necrosis $(43.0 \%)$. These data underline the hypothesis that POAF is frequently associated with postoperative complications and is likely a systemic manifestation of a local complication. The median time interval between the onset of POAF and diagnosis of anastomotic leakage was 3 days. Hence, POAF might trigger the medical team to carry out an early investigation to diagnose infectious complications. Diltiazem prophylaxis might not prevent POAF in all cases because a different grade of systemic inflammation in infectious complications potentially overrules the antiarrhythmic effect of diltiazem. In contrast, successful pharmacologic prophylaxis for POAF in esophagectomy might mask a warning sign and subsequently delay the diagnosis and treatment of anastomotic leaks. However, this effect was not evident in our data.
Overall, preventing POAF with a single drug that affects a single pathway seems unlikely because the etiology of POAF is multifactorial and its mechanism of action is incompletely understood. To prevent POAF in clinical practice, the focus should be on control of preventable perioperative risk factors: electrolyte imbalances, perioperative hypervolemia/hypovolemia, hypotension and anemia [44]. Moreover, we revealed that most episodes of POAF arose in the setting of anastomotic and septic complications. Consequently, an optimal technical outcome is important. Technical improvements like ischemic conditioning of the gastric conduit before esophagectomy [45] as well as intraoperative indocyanine fluorescence are promising and have been reported to improve tissue perfusion. The latter still needs randomized, multicenter trials to proof the benefit [46]. Additionally, minimal invasive procedures might reduce the perioperative trauma. Still, these improvements are limited by the anatomy of the patient and up to now anastomotic leakage remains unchanged. From the technical point of view, it would be conceivable that the site of anastomosis matters. There are data that cervical and thoracic anastomosis are equally safe [47, 48], but there are also data that intrathoracical anastomosis leads to less leakage and wound infection [49]. Promising results have been reported of enhanced recovery protocols for esophagectomy. A systematic review and pooled analyses showed a reduced incidence of anastomotic leak for patients followed by an enhanced recovery protocol compared to those undergoing esophagectomy followed by a usual care [50]. Alternative surgical approaches combining laparoscopy with thoracotomy or, if feasible, a completely minimally invasive procedure lead to reduce pulmonary complications but did not change the number of anastomotic leakage [51].

In case of a suspected leakage, early detection of infectious complications by clinical examination, laboratory studies to screen for signs of infection (e.g., increased leukocyte count, procalcitonin level or CRP level) and subsequent CT of the chest and abdomen and/or endoscopy (if appropriate) is necessary.

Our study had several limitations. It was a single-center, retrospective analysis, and there was a selection bias because many patients with thoracoabdominal esophagectomy were excluded. An advantage of our design was the comparability of study participants with regard to perioperative regimens. All data were derived from an in-patient routine database of our hospital and were not collected specifically for our study. Only documented cases of POAF were taken into account, which may have underestimated the true prevalence of POAF. Furthermore, patients may not have reported outpatient episodes of AF to their general practitioner. Hence, these outcomes were missing and would have led to incorrect estimation of the prevalence of 
POAF in this population. Stawicki et al. [52] reported the onset of POAF to be $\sim 90 \%$ within POD3 after esophagectomy. Thus, the number of missed POAFs after hospital discharge is probably low.

\section{Conclusions}

In this retrospective analysis, a prophylactic 14-day postoperative course of diltiazem was not associated with a reduction in the prevalence of new-onset AF or 30-day mortality following thoracoabdominal esophagectomy. Prophylactic diltiazem was associated with drug-related adverse effects such as bradycardia and increased requirement of norepinephrine. Consequently, the prophylactic effect of diltiazem is questionable, and intensivists should carefully weigh the risk versus theoretical benefit of diltiazem in patients after thoracoabdominal esophagectomy. POAF seldom occurs without complications, so it could function as an early warning sign for anastomotic leaks and may, thus, be of clinical importance.

Based on the results of this study, we no longer use diltiazem prophylaxis as standard care for these patients. Instead, we focus on control of preventable perioperative risk factors and introduced an enhanced recovery protocol.

Acknowledgements Open Access funding provided by Projekt DEAL. We thank B. Lnenicka, MD, for her assistance with data collection and for comments that improved the manuscript.

Author's contribution $\mathrm{MH}$ and CJB involved in study conception and design, acquisition of data, analysis and interpretation of data, drafting of manuscript; LS and TS took part in interpretation of data, drafting of manuscript; BHS participated in analysis and interpretation of data, drafting of manuscript; TB took part in statistics, analysis and interpretation of data, critical revision of manuscript. BPM, MKD, KS, MWB and MAW involved in interpretation of data, critical revision of manuscript. All authors gave final approval of the version to be submitted.

Funding This study was carried out with financial resources of the Department of Anesthesiology and Intensive Care Medicine (University of Heidelberg, Germany) and the Department of General, Visceral and Transplantation Surgery (University of Heidelberg, Germany).

\section{Compliance with ethical standards}

Conflict of interest The authors declare that they have no competing interest.

Open Access This article is licensed under a Creative Commons Attribution 4.0 International License, which permits use, sharing, adaptation, distribution and reproduction in any medium or format, as long as you give appropriate credit to the original author(s) and the source, provide a link to the Creative Commons licence, and indicate if changes were made. The images or other third party material in this article are included in the article's Creative Commons licence, unless indicated otherwise in a credit line to the material. If material is not included in the article's Creative Commons licence and your intended use is not permitted by statutory regulation or exceeds the permitted use, you will need to obtain permission directly from the copyright holder. To view a copy of this licence, visit http://creativecommons. org/licenses/by/4.0/.

\section{References}

1. Colwell EM, Encarnacion CO, Rein LE et al (2018) Atrial fibrillation after transhiatal esophagectomy with transcervical endoscopic esophageal mobilization: one institution's experience. J Cardiothorac Surg 13:73

2. Irshad K, Feldman LS, Chu VF et al (2002) Causes of increased length of hospitalization on a general thoracic surgery service: a prospective observational study. Can J Surg 45:264-268

3. Joshi KK, Tiru M, Chin T et al (2015) Postoperative atrial fibrillation in patients undergoing non-cardiac non-thoracic surgery: a practical approach for the hospitalist. Hosp Pract 43(1995):235-244

4. Ivanovic J, Maziak DE, Ramzan S et al (2014) Incidence, severity and perioperative risk factors for atrial fibrillation following pulmonary resection. Interact CardioVasc Thorac Surg 18:340-346

5. Bhave PD, Goldman LE, Vittinghoff E et al (2012) Incidence, predictors, and outcomes associated with postoperative atrial fibrillation after major noncardiac surgery. Am Heart $\mathrm{J}$ 164:918-924

6. Gialdini G, Nearing K, Bhave PD et al (2014) Perioperative atrial fibrillation and the long-term risk of ischemic stroke. JAMA 312:616-622

7. Frendl G, Sodickson AC, Chung MK et al (2014) 2014 AATS guidelines for the prevention and management of perioperative atrial fibrillation and flutter for thoracic surgical procedures. J Thorac Cardiovasc Surg 148:e153-e193

8. Murthy SC, Law S, Whooley BP et al (2003) Atrial fibrillation after esophagectomy is a marker for postoperative morbidity and mortality. J Thorac Cardiovasc Surg 126:1162-1167

9. Sabel MS, Smith JL, Nava HR et al (2002) Esophageal resection for carcinoma in patients older than 70 years. Ann Surg Oncol 9:210-214

10. Vaporciyan AA, Correa AM, Rice DC et al (2004) Risk factors associated with atrial fibrillation after noncardiac thoracic surgery: analysis of 2588 patients. J Thorac Cardiovasc Surg 127:779-786

11. Dixit S (2009) Atrial fibrillation after major thoracic surgery: new insights into underlying mechanisms. J Am Coll Cardiol 54:2049-2051

12. Echahidi N, Pibarot P, O'Hara G et al (2008) Mechanisms, prevention, and treatment of atrial fibrillation after cardiac surgery. J Am Coll Cardiol 51:793-801

13. Kowey PR, Taylor JE, Rials SJ et al (1992) Meta-analysis of the effectiveness of prophylactic drug therapy in preventing supraventricular arrhythmia early after coronary artery bypass grafting. Am J Cardiol 69:963-965

14. Zimetbaum $P$ (2007) Amiodarone for atrial fibrillation. N Engl J Med 356:935-941

15. Amar D, Roistacher N, Rusch VW et al (2000) Effects of diltiazem prophylaxis on the incidence and clinical outcome of atrial arrhythmias after thoracic surgery. $\mathrm{J}$ Thorac Cardiovasc Surg 120:790-798

16. Van Mieghem W, Tits G, Demuynck K et al (1996) Verapamil as prophylactic treatment for atrial fibrillation after lung operations. Ann Thorac Surg 61:1083-1085 discussion 1086 
17. Lindgren L, Lepantalo M, von Knorring J et al (1991) Effect of verapamil on right ventricular pressure and atrial tachyarrhythmia after thoracotomy. Br J Anaesth 66:205-211

18. Sedrakyan A, Treasure T, Browne J et al (2005) Pharmacologic prophylaxis for postoperative atrial tachyarrhythmia in general thoracic surgery: evidence from randomized clinical trials. J Thorac Cardiovasc Surg 129:997-1005

19. Echizen H, Eichelbaum M (1986) Clinical pharmacokinetics of verapamil, nifedipine and diltiazem. Clin Pharmacokinet 11:425-449

20. Barreto JC, Posner MC (2010) Transhiatal versus transthoracic esophagectomy for esophageal cancer. World J Gastroenterol 16:3804-3810

21. Heger P, Blank S, Goossen K et al (2019) Thoracoabdominal versus transhiatal surgical approaches for adenocarcinoma of the esophagogastric junction-a systematic review and meta-analysis. Langenbeck's Arch Surg 404:103-113

22. Blank S, Schmidt T, Heger P et al (2018) Surgical strategies in true adenocarcinoma of the esophagogastric junction (AEG II): thoracoabdominal or abdominal approach? Gastric Cancer 21:303-314

23. Schaible A, Brenner T, Hinz U et al (2017) Significant decrease of mortality due to anastomotic leaks following esophageal resection: management makes the difference. Langenbeck's Arch Surg 402:1167-1173

24. Hochreiter M, Uhling M, Sisic L et al (2018) Prolonged antibiotic prophylaxis after thoracoabdominal esophagectomy does not reduce the risk of pneumonia in the first 30 days: a retrospective before-and-after analysis. Infection 46:617-624

25. Tisdale JE, Wroblewski HA, Wall DS et al (2010) A randomized, controlled study of amiodarone for prevention of atrial fibrillation after transthoracic esophagectomy. J Thorac Cardiovasc Surg 140:45-51

26. McCormack O, Zaborowski A, King S et al (2014) New-onset atrial fibrillation post-surgery for esophageal and junctional cancer: incidence, management, and impact on short- and longterm outcomes. Ann Surg 260:772-778 discussion 778

27. Reiffel JA, Verma A, Kowey PR et al (2017) Incidence of previously undiagnosed atrial fibrillation using insertable cardiac monitors in a high-risk population: the REVEAL AF study. JAMA Cardiol 2:1120-1127

28. Lohani KR, Nandipati KC, Rollins SE et al (2015) Transthoracic approach is associated with increased incidence of atrial fibrillation after esophageal resection. Surg Endosc 29:2039-2045

29. Okamura A, Takeuchi H, Matsuda S et al (2015) Factors affecting cytokine change after esophagectomy for esophageal cancer. Ann Surg Oncol 22:3130-3135

30. Wu N, Xu B, Xiang Y et al (2013) Association of inflammatory factors with occurrence and recurrence of atrial fibrillation: a meta-analysis. Int J Cardiol 169:62-72

31. Tisdale JE, Wroblewski HA, Wall DS et al (2009) A randomized trial evaluating amiodarone for prevention of atrial fibrillation after pulmonary resection. Ann Thorac Surg 88:886-893 discussion 894-885

32. Bayliff CD, Massel DR, Inculet RI et al (1999) Propranolol for the prevention of postoperative arrhythmias in general thoracic surgery. Ann Thorac Surg 67:182-186

33. Ojima T, Nakamori M, Nakamura M et al (2017) Randomized clinical trial of landiolol hydrochloride for the prevention of atrial fibrillation and postoperative complications after oesophagectomy for cancer. Brit J Surg 104:1003-1009
34. Tisdale JE, Jaynes HA, Watson MR et al (2019) Amiodarone for prevention of atrial fibrillation following esophagectomy. J Thorac Cardiovasc Surg 158:301.e301-310.e301

35. Jakobsen CJ, Bille S, Ahlburg P et al (1997) Perioperative metoprolol reduces the frequency of atrial fibrillation after thoracotomy for lung resection. J Cardiothorac Vasc Anesth 11:746-751

36. Amar D, Roistacher N, Burt ME et al (1997) Effects of diltiazem versus digoxin on dysrhythmias and cardiac function after pneumonectomy. Ann Thorac Surg 63:1374-1381 discussion 1381-1372

37. Fumagalli U, Melis A, Balazova $J$ et al (2016) Intra-operative hypotensive episodes may be associated with post-operative esophageal anastomotic leak. Updates Surg 68:185-190

38. Soward AL, Vanhaleweyk GL, Serruys PW (1986) The haemodynamic effects of nifedipine, verapamil and diltiazem in patients with coronary artery disease. A review. Drugs 32:66-101

39. O'Connor SE, Grosset A, Janiak P (1999) The pharmacological basis and pathophysiological significance of the heart ratelowering property of diltiazem. Fundam Clin Pharmacol 13:145-153

40. Loick HM, Schmidt C, Van Aken H et al (1999) High thoracic epidural anesthesia, but not clonidine, attenuates the perioperative stress response via sympatholysis and reduces the release of troponin $\mathrm{T}$ in patients undergoing coronary artery bypass grafting. Anesth Analg 88:701-709

41. Kirchhof P, Benussi S, Kotecha D et al (2016) 2016 ESC Guidelines for the management of atrial fibrillation developed in collaboration with EACTS. Eur Heart J 37:2893-2962

42. January CT, Wann LS, Alpert JS et al (2014) 2014 AHA/ACC/ HRS guideline for the management of patients with atrial fibrillation: executive summary: a report of the American College of Cardiology/American Heart Association Task Force on practice guidelines and the Heart Rhythm Society. Circulation 130:2071-2104

43. Seesing MFJ, Scheijmans JCG, Borggreve AS et al (2018) The predictive value of new-onset atrial fibrillation on postoperative morbidity after esophagectomy. Dis Esophagus 31:doy028

44. Bessissow A, Khan J, Devereaux PJ et al (2015) Postoperative atrial fibrillation in non-cardiac and cardiac surgery: an overview. J Thromb Haemost 13(Suppl 1):S304-S312

45. Bludau M, Holscher AH, Vallbohmer D et al (2010) Ischemic conditioning of the gastric conduit prior to esophagectomy improves mucosal oxygen saturation. Ann Thorac Surg 90:1121-1126

46. Ladak F, Dang JT, Switzer N et al (2019) Indocyanine green for the prevention of anastomotic leaks following esophagectomy: a meta-analysis. Surg Endosc 33:384-394

47. Walther B, Johansson J, Johnsson F et al (2003) Cervical or thoracic anastomosis after esophageal resection and gastric tube reconstruction: a prospective randomized trial comparing sutured neck anastomosis with stapled intrathoracic anastomosis. Ann Surg 238:803-812 discussion 812-804

48. Liu YJ, Fan J, He HH et al (2018) Anastomotic leakage after intrathoracic versus cervical oesophagogastric anastomosis for oesophageal carcinoma in Chinese population: a retrospective cohort study. BMJ Open 8:e021025

49. Klink CD, Binnebosel M, Otto J et al (2012) Intrathoracic versus cervical anastomosis after resection of esophageal cancer: a matched pair analysis of 72 patients in a single center study. World J Surg Oncol 10:159 
50. Markar SR, Karthikesalingam A, Low DE (2015) Enhanced recovery pathways lead to an improvement in postoperative outcomes following esophagectomy: systematic review and pooled analysis. Dis Esophagus 28:468-475

51. Mariette C, Markar SR, Dabakuyo-Yonli TS et al (2019) Hybrid minimally invasive esophagectomy for esophageal cancer. N Engl J Med 380:152-162
52. Stawicki SP, Prosciak MP, Gerlach AT et al (2011) Atrial fibrillation after esophagectomy: an indicator of postoperative morbidity. Gen Thorac Cardiovasc Surg 59:399-405

Publisher's Note Springer Nature remains neutral with regard to jurisdictional claims in published maps and institutional affiliations. 